\title{
Stem Cell Lineages: Between Cell and Organism
}

\author{
Melinda Bonnie Fagan*
}

Ontologies of living things are increasingly grounded on the concepts and practices of current life science. Biological development is a process, undergone by living things, which begins with a single cell and (in an important class of cases) ends with formation of a multicellular organism. The process of development is thus prima facie central for ideas about biological individuality and organismality. However, recent accounts of these concepts do not engage developmental biology. This paper aims to fill the gap, proposing the lineage view of stem cells as an ontological framework for conceptualizing organismal development. This account is grounded on experimental practices of stem cell research, with emphasis on new techniques for generating biological organization in vitro. On the lineage view, a stem cell is the starting point of a cell lineage with a specific organismal source, time-interval of existence, and 'tree topology' of branch-points linking the stem to developmental termini. The concept of 'enkapsis' accommodates the cell-organism relation within the lineage view; this hierarchical notion is further explicated by considering the methods and results of stem cell experiments. Results of this examination include a (partial) characterization of stem cells' developmental versatility, and the context-dependence of developmental processes involving stem cells.

\section{Keywords}

biological individuality $\bullet$ cell-organism relation $\bullet$ development $\bullet$ stem cells $\bullet$ lineage model experiment $\bullet$ organoids

Part of a special issue, Ontologies of Living Beings, guest-edited by A. M. Ferner and Thomas Pradeu

Editorial introduction: In this contribution, Melinda Fagan asks what stem cells are, what they tell us about the idea of biological development, and how they influence our concepts organism and biological individual. 'Stem cells' are cells that can self-renew and differentiate into more specialized cells. In the last fifteen years or so, they have been at centre of research into regenerative medicine.

Fagan's piece is strongly interdisciplinary. First, she combines two, typically distant, biological domains - namely stem cell biology and systematics. She shows how Hennig's

*Department of Philosophy, Carolyn Tanner Irish Humanities Bldg 215, S Central Campus Dr, 4th Floor, Salt Lake City, UT, USA, mel.fagan@utah.edu

Received 2 September 2016; Accepted 20 February 2017

doi:10.3998/ptb.6959004.0009.006 
concept of an 'enkaptic system' (a concept pertaining to systematics, which describes the encapsulation of systems of lower complexity into systems of higher complexity) can shed light on the lineage model of stem cells.

Second, Fagan connects this biological perspective with long-standing metaphysical issues about the definition of biological individuals and organisms. She shows that development has been unduly neglected in philosophical debates on the concept of a biological individual. For her, a multicellular organism could be conceived as the product of stem cells' enkaptic lineages, which realize some or all of a variety of modes of development ('normal,' 'pathological,' 'embryo-like,' etc.)—themselves reflected in various experimental settings. This places development (and more precisely a stem cell-based view of development) at the heart of the concept of an organism. Fagan adds that, contrary to traditional views of development as 'autonomous' and 'deterministic,' stem cell-initiated development is in fact context-dependent and reversible. This constitutes the basis for a novel view of the building of organisms and of the conditions of their spatio-temporal identity.

The paper offers detailed analyses of current stem cell biology and the scientific practices of this domain. From this point of view, Fagan's work illustrates a recent trend emphasizing the importance of practical and experimental approaches to metaphysical issues. However, the paper also raises insightful questions for more traditionally-oriented metaphysicians. For instance, could the idea that stem cells are 'future-oriented' be part of a teleological approach to the living world-and if so in what sense of 'teleology'? If stem cell-initiated development is flexible, reversible, and not internally driven, as Fagan suggests, will it still be legitimate to talk of a teleological view? Moreover, what does it mean to say that stem cells are defined by their 'potency' (and their degrees of potency, from totipotent to unipotent stem cells)? It would be particularly interesting to know whether Aristotelian scholars might consider the 'potentiality' and 'future-orientation' of stem cells as modern avatars of Aristotle's views (on modern remnants of teleology — for which, see also the contribution of Christopher Shields in this special issue).

$-\mathrm{AF} / \mathrm{TP}$

\section{Introduction}

There has been much recent discussion of biological individuality and the nature of organisms in philosophy of biology (e.g. Dupré and O'Malley 2009; Godfrey-Smith 2009; Pradeu 2012; Bouchard and Huneman 2013; Guay and Pradeu 2016; Chen et al. under review). These recent ontological discussions tend to be critical of earlier accounts as not including the full diversity of living things: microorganisms, plants, colonial species, symbiotic associations, viruses, and more. Yet the most recent proposed ontologies of living things share with their predecessors a tendency to privilege evolutionary theory and genetics at the expense of other areas of biology. The bias is unsurprising, as those two fields are disproportionately the focus of philosophy of biology in general (Pradeu 2016). But, just as philosophers have tended to underestimate the diversity of the living world, they have tended to overlook certain areas of biology when considering ontological questions. One such area is developmental biology, the field aimed at understanding processes of change in organisms from birth to death. 1 These processes are, on the face of it, central to questions of biological individuality and organismality. Every organism

\footnotetext{
${ }^{1}$ There is in fact some dispute as to the boundaries of the process of development; i.e., whether it is complete at maturity or continues until death (see Pradeu et al. 2011, thematic section on 'The boundaries of development'). Although I favor the latter answer, the claims of this paper do not depend on that assumption.
} 
undergoes changes over its lifetime. The subset of those changes that are directional and robust across members of a species together comprise the phenomena of development. The capacity to develop, to undergo a directed and robust process of change that repeats in successive generations, is a characteristic feature of organisms. In other words, organisms are the biological individuals that develop. In multicellular organisms, development includes the transformation of a single cell (the zygote) to a multicellular embryo and then to a mature organism. The developing entity, while remaining in some sense a single individual, transitions from the cellular to multicellular level of biological organization.

Recent accounts of biological individuality and organismality, however, tend to elide the process of development and the transformation from cell to organism. For example, Clarke (2010) surveys 13 proposed criteria of biological individuality: reproduction/persistence, life cycle, genetics, sex, bottleneck, germ-soma separation, policing mechanisms, spatial boundaries, histocompatibility, fitness maximization, cooperation and conflict, codispersal, and adaptations. Development, if included at all, is relegated to an aspect of the life cycle or reduced to the germ-soma distinction; bottleneck, reproduction, and genetic criteria sidestep developmental processes entirely. Clarke's approach represents the received view in philosophy of biology, that the role of individuality concepts in evolutionary theory suffices to answer ontological questions about biological individuality more generally (313-314). But evolutionary theory is not unique in finding the concept of the individual 'indispensable'. As Clarke also notes, '[b]iological individuals are the entities that develop in ontogeny,' just as they are the entities that differentially survive and reproduce in evolution (313). Studies of biological development have just as much claim to significance for ontology as studies of evolution.

Pradeu (2012) and colleagues reject the received view, articulating and defending a 'physiological' account of biological individuality grounded in immunology. But the prevalence of evolutionary ideas in philosophy of biology frames even this project, which lumps together many non-evolutionary fields of biology as 'physiological'-biochemistry, molecular biology, cell biology, developmental biology, immunology, and more. This is taken by many philosophers (including Clarke, cited above) to mean that physiological approaches to biological individuality can all be considered together in contrast to evolutionary accounts, diluting the importance of distinct fields such as developmental biology. Similarly, Dupré and O'Malley (2009) critically examine common criteria for entities to be classified as 'living': spatial boundedness, metabolism, reproduction, evolvability. Again, development is effaced as a feature of living things. Dupré and O'Malley do not endorse these criteria, but instead propose an alternative ontology, based on the idea that living things distinctively exhibit autonomy through interactive collaboration, such that 'the entities that we primarily think of as organisms, are in fact cooperating assemblies of a wide variety of lineage-forming entities ... the intersection of lineages and metabolism' (Dupré 2012). Although this ontological approach is relevant to developmental processes, Dupré and O'Malley do not consider them, focusing instead on the origin of life, symbiosis, and 'entities that form reproducing and evolving lineages'-i.e., topics related to evolutionary biology (ibid). On the whole, lack of attention to developmental processes impoverishes current ontological accounts of living things.

${ }^{2}$ Multicellular organisms are in the minority of living things (O'Malley 2014; Minelli and Pradeu 2014). However, the problem of multicellular development remains significant for ontology, even if it is not universal. Minelli (2011) also argues against the necessity of a single-cell starting point for multicellular development, citing asexual budding. Although this paper does not address such cases directly, the model of stem cell-mediated development set out below has resources to accommodate them. I will examine these issues in a future paper. 
This paper follows the general approach of Pradeu, Dupré, O’Malley, and others in looking to areas of biology beyond those traditionally considered by philosophers as relevant to the ontology of living things. I follow Dupré and O'Malley somewhat further in emphasizing lineages. However, the lineages discussed below are not evolving lineages of organisms, but of dividing and differentiating cells that constitute a developing multicellular organism. The field of life science most centrally concerned with the transformation from single cell to multicellular organism is stem cell biology. A stem cell is 'functionally defined as having the capacity to selfrenew and the ability to generate differentiated cells' (Melton 2013, 7). ${ }^{3}$ That is, a stem cell is a cell that can divide to produce offspring that are also stem cells, and produce descendants that are specialized for particular functions within a developed organism. 4 Stem cells so defined mediate the developmental transition between cell and organismal levels for multicellular organisms. In previous work, I have articulated an abstract framework for clarifying the main concepts, methods, results and aims of stem cell biology today (Fagan 2013a). This paper elaborates that conceptual framework, to fill the gap in our current accounts of biological individuality and organismality.

The basic idea of this framework, to anticipate the main claims articulated and defended below, is that a stem cell is the starting point of a cell lineage with a specific organismal source, time-interval of existence, and 'tree topology' of branch-points linking the stem to developmental termini (Section 2). This lineage view is grounded on experimental practices of stem cell research, interpreted in terms of concepts that figure prominently in that science. This approach to ontological questions is evidently unlike that of traditional analytic metaphysics. An ontology for biological development, on the 'scientific practice-based' approach taken here, is a conceptual framework for understanding that process, and as such should be closely connected to the branches of inquiry that have this aim. Although stem cell research does not subsume the totality of studies of biological development, as a field with rich interdisciplinary ties and resources it does connect with the greater part of them. It is a further question, beyond the scope of this paper, as to how the lineage view might connect with ontologies of biological development articulated using other approaches, such as traditional analytic metaphysics. Answers to this further question are one hoped-for outcome of the present Special Issue. However, the particular ontology proposed here, the lineage view, exhibits some overlap with ontologies grounded in quite different areas of biology: phylogenetics and systematics (Section 3). This offers the prospect of a unified ontology of living things. A further result of considering experimental practices in stem cell biology is insight into the transition between cell and organismal levels. Of particular interest here are two new experimental products of stem cell research: stem cell-derived organoids and embryo-like spatial patterning. These, together with more estab-

\footnotetext{
${ }^{3}$ See also the glossaries of the International Society for Stem Cell Research (2016) and the European Stem Cell Network (2016), the Cell Therapy and Regenerative Medicine Glossary (2012), National Institutes of Health Stem Cell Glossary (2015), and further references in Fagan 2013a. The term 'stem cell' has had a number of meanings since its introduction as the Anglicization of Haeckel's Stamzelle (Dröscher 2014). The ontology proposed here is premised on the currently-prevalent general scientific definition of 'stem cell.'

${ }^{4}$ The term 'self-renewal' is thus somewhat misleading; stem cells do not literally generate themselves through cell division. Rather, they produce offspring cells that are classified as stem cells of the same kind.

${ }^{5}$ I have discussed some aspects of the lineage view in earlier work (Fagan 2013a, 2013b, 2016a, under review $b$ ). These previous treatments focus primarily on the epistemology of stem cell research, however: evidence for hypotheses about stem cell capacities, explanatory models, modeling relations in stem cell experiments.
} 
lished experimental practices, clarify the concept of developmental potential as it bears on the cell-organism transition (Section 4). From consideration of these experimental practices and their products, I explicate the notion of developmental versatility, and draw some conclusions about biological individuality and organismality (Section 5). Section 6 concludes.

\section{The Lineage Model}

First, some background. A stem cell is not a special kind of single, isolated cell. There are widelyaccepted and unambiguous criteria for individuating single cells: each single cell is discretely bounded by an enclosing membrane, within which there are distinguishable parts (organelles) that sustain that cell's survival and various behaviors. There are also clear 'rules' for individuating cells across generations, articulated as tenets of Cell Theory. Cells reproduce by binary division; a parent cell divides to produce two offspring cells. 6 When cells reproduce by division, the process is finished when the membranes 'pinch off' to separate. An individual cell's existence begins with a cell division event and ends with either a second division event (producing two offspring) or cell death (and no offspring). Generations of cells linked by reproductive division form a lineage (Figure 1). A lineage in general is a complex biological entity, extending over multiple generations of other, lower-level entities. In cell lineages, the lower-level entities are

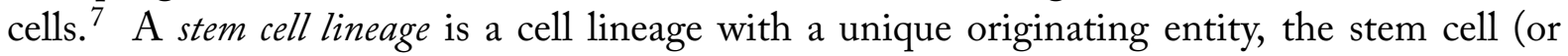
stem cell population). To be a stem cell is to be the origin of a cell lineage, either actually or potentially. Another way to put the point is that stem cells are essentially generative: they can produce other cells, both like and unlike themselves.

This is why stem cells are not just individual cells that share certain other properties. Stem cells are not individual 'units of life,' each separated from its environment by a bounding membrane. Rather, a stem cell is the starting point of a cell lineage; it is a cell defined by its reproductive abilities rather than by standard parts and an enclosing membrane. The concept of a stem cell involves multiple generations, because to self-renew is to produce either one or two cells resembling the parent, which ceases to exist upon splitting. Because they are defined in terms of their descendants, stem cells are essentially future-oriented. Moreover, the future that stem cells point toward includes developmental transformation. This future-oriented aspect is conceptualized as stem cells' potential or potency. Every stem cell (or type of stem cell) has a characteristic potency or potential: i.e., the range of cell types to which that stem cell can give rise. The maximum developmental potential is totipotency: the capacity to produce an entire organism (and, in mammals, extra-embryonic tissues). Pluripotency is the ability to produce all cell types of an adult organism. More restricted stem cells are multipotent: able to produce some, but not all, mature cell types. Stem cells that can give rise to only a few mature cell types are oligopotent. Minimum differentiation potential is unipotency: the capacity to produce only single cell type. So the concept of potential, or potency, provides a rough ordering of stem cell types in terms of their developmental range.

Importantly, the ordering of stem cell potencies implicates both cellular and organismal levels of organization. Different categories of stem cell potential qualitatively refer to the number of mature cell types that can be generated from a given 'stem' (multi-, oligo-, uni-). But the

\footnotetext{
${ }^{6}$ Some organisms produce gamete cells, which can also fuse to form a new cell: a zygote. Although a zygote could be considered a stem cell (and has been; see Dröscher 2014), this is not how the term is used today. So meiosis and fertilization can be set aside, for the purpose of this paper.

${ }^{7}$ The component cells of a lineage can be individual cells or cell populations; the lineage framework applies to both.
} 


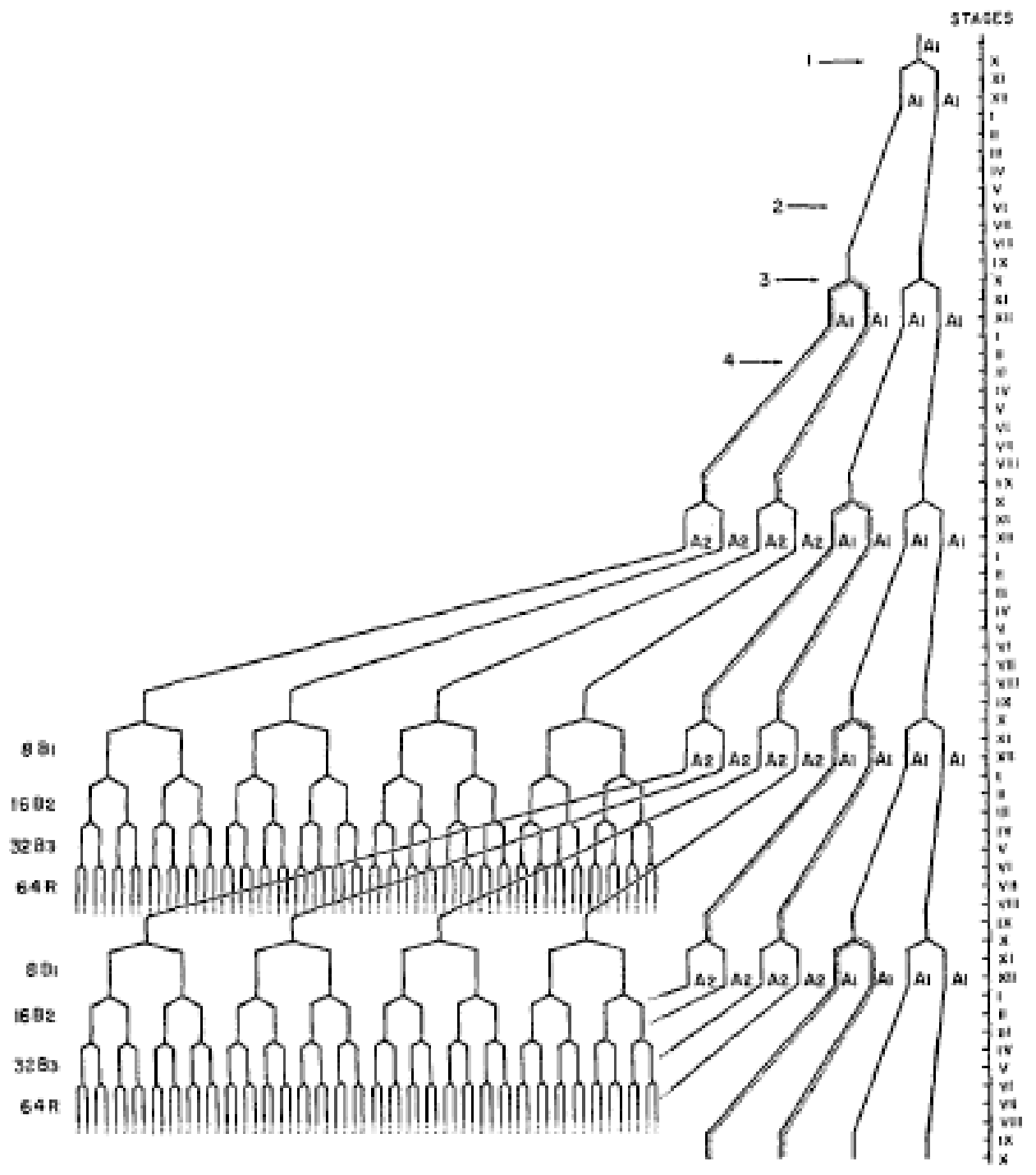

Fig. 14 Detalled diagram representing the evolution of the progeny of one type $\mathbf{A}_{1}$ eell under seheme 4 . The diagram eneompasses 5 eonsecutive eyeles of the seminiferous epithelium. The two eycles at the base of the diagram show the behavior of all spermatogonial types. Arrows 1-4 show 4 possible sites for the initiation of differentiation.

Figure 1: Diagram of a lineage produced by cell division (from Clermont and Leblond 1959, 267; reprinted with permission from John Wiley and Sons, Inc.). 
categories also reference the whole organism. The distinction between pluri- and multipotency is drawn in terms of the cell types composing the body of a mature organism (all or some, respectively), while the distinction between toti- and pluripotency is between levels of organization: a whole organismal body, contrasted with all the cell types that make up that body. ${ }^{8}$ This involvement of the organismal level, I shall argue, is crucial to the concept of a stem cell. For now, the point I want to emphasize is that the very idea of a stem cell involves a cell lineage.

Conditions of existence for a stem cell lineage can be expressed as four elements:

1. origin: the multicellular organism from which the stem cell is derived;

2. interval of persistence: number and rate of self-renewing stem cell divisions;

3. component traits: characters of cells occupying the various positions in a lineage;

4. process of change: stages of the lineage are ordered by a developmental process.

I discuss each briefly.

(1) Origin: every stem cell is a part of, or descended via cell division from a part of, exactly one multicellular organism. Scientific names for different varieties of stem cell often refer to features of this organism-notably, species and developmental stage. For example, 'hESC' is an abbreviation of 'human embryonic stem cell,' and 'm-epiSC' of 'mouse epiblast stem cell'. Stem cells found within a mature organism are named for the part of that organism they build and/or replenish: neural stem cells, hematopoietic (blood-forming) stem cells, epithelial stem cells, muscle stem cells, and so on. More broadly, stem cell research has long been divided into two branches, which focus on 'adult' or 'embryonic' stem cells. The adult/embryonic distinction refers to the developmental stage of stem cells' organismal source. For identifying stem cells, the key characters of an organismal source are its species, developmental stage, and the location from which stem cells are derived.

(2) Persistence: like other biological entities, stem cell lineages have finite lifespans. The lifespan of a stem cell lineage is constrained by the duration of the initiating stem cell's self-renewal capacity. This duration varies across different types of stem cell, although for cultured stem cell lines there appears to be no upper limit. When self-renewal ceases, the stem cell population will steadily deplete through differentiation and cell death, followed by the descendant populations.

(3) Cell traits: These descendant populations are distinguished from stem cells and from one another by a set of variable characters $C_{1}, \ldots, C_{n}$. In this sense, the distribution of values for characters $C$ organizes a stem cell lineage, distinguishing cells that occupy different positions within that lineage.

(4) Process: character-values for $C$ map onto a developmental process $D$, which orders the different cell states appearing in the lineage $\left(s_{1}, \ldots, s_{n}\right)$. I further analyze this process, and associated characters $C$, below.

These four elements correspond to variables that can take different values, corresponding to the different varieties of stem cell $\left(L_{1}, L_{2}, L_{3}, n, C_{1}, \ldots, C_{n}, D, s_{1}, \ldots, s_{n}\right)$. 9 Taken together, this framework of variables comprises the lineage view of stem cells. Values of these variables are specified by the materials and methods of experiments aimed at identifying and characterizing a particular kind of stem cell. These experiments share a common basic design. First, cells

${ }^{8}$ Cell types are the specialized building blocks of multicellular organisms, such as neurons, fibroblasts (including but not limited to skin cells), muscle cells, and so on. Different cell types robustly exhibit different molecular, biochemical, morphological, and functional characteristics. It is in virtue of having these features that non-stem cells are termed 'specialized.' Cells that have these features stably, such that no more developmental transformation occurs within or across generations, are terminally differentiated.

${ }^{9}$ See Fagan 2013a for more detail about this feature of the lineage view. 
are removed from an organismal source and placed in a context in which their character-values can be measured. After the measurements are made, some of these cells are moved to a new environment, where development can proceed. Lastly, character-values of resulting developed cells are measured. By this procedure, the model's variables are specified, and experimental results correlate character values of the organismal source, candidate stem cells, and developed cells.

To identify a stem cell is to characterize its two defining capacities: self-renewal and differentiation potential. This characterization corresponds to a stem cell lineage exhibiting a particular structure, or topology. Lineages in general are modeled using 'tree diagrams' that track relations between generations of reproducing entities (Figure 2). The 'tree topology' framework leads to further analysis of the developmental process that orders cell states in a stem cell lineage (variable $D$ ). On this approach, variable $D$ corresponds to a space of possible tree-models. Each model within this space has a particular number and arrangement of developmental stages, branch-points, and termini. The simplest such model is a linear sequence of cell states ordered in time. However, cell developmental processes are coordinated with cell division events, such that stages can form a branching hierarchy rather than a single linear sequence. The number of stages determines the depth of cell hierarchy. The arrangement of branch-points (bi-, tri- or $\mathrm{n}$-furcations) indicates the arrangement of distinct developmental pathways initiated by a stem cell. The number of termini corresponds to a stem cell's developmental potential.

The way branch-points are distributed among the stages leading from a stem cell state to developmental termini determines a particular tree topology. The simplest cases, like asymmetric cell division, distinguish only two stages: a stem and a more differentiated state (Figure 2, top left). Other developmental processes are more elaborate (Figure 2, bottom). Some varieties of stem cell, such as those for skin and hair, initiate developmental processes with multiple stages but few branch-points. In contrast, stem cells of the blood and immune system form elaborate branching hierarchies. So we can characterize a cell developmental process $D$ (an arrangement of cell states $s_{1}, \ldots, s_{n}$ ) in terms of the characters of the corresponding tree topology. This elaborated lineage framework offers a general way to compare different varieties of stem cell. Any given process of development produces a cell lineage tree with a specific topology, representing developmental and reproductive relations between cells. Different stem cell concepts correspond to models that differentially constrain the space of possible topologies for cell lineage trees. In this lineage framework, stem cell identity has parallels with phylogenetic trees in systematics. The next section explores these parallels, with respect to the cell-organism relation.

\section{Ontogeny and Phylogeny: Stem Cell Lineages as Enkaptic Systems}

Stem cell potential is coupled to the process of organismal development, in that stem cells from early developmental stages of an organism (e.g., pre-implantation mammalian embryos) have wider potential than stem cells from later developmental stages (e.g., fetuses, adults). So stem cells have a mutually generative relationship with multicellular organisms; they are both source and result of organismal organization.10 The lineage model as presented so far, however, does not address the latter. The remaining sections elaborate the lineage model so as to include the transition between cell and organism. The first step is to extend the lineage view by borrowing an idea from systematics: Hennig's concept of an enkaptic system. ${ }^{11}$ The term 'enkapsis' derives

\footnotetext{
${ }^{10} \mathrm{~A}$ generative cycle of organism-stem cell-organism does not require organismal reproduction, although the two processes are compatible.

${ }^{11}$ This idea was suggested by Matt Haber, and follows recent work by Rieppel (2009).
} 

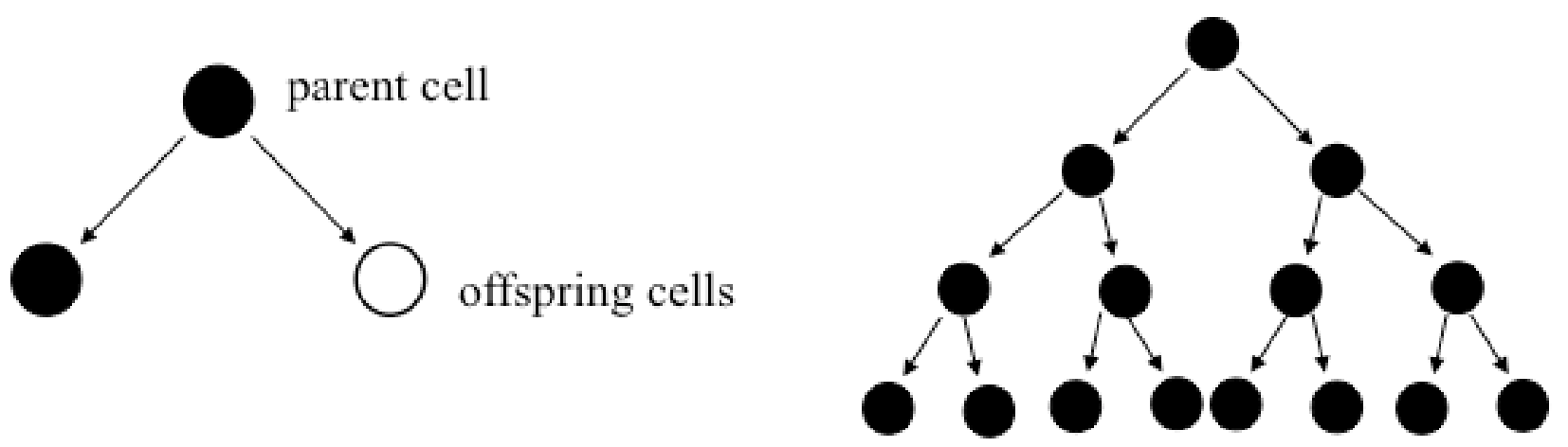

(a)

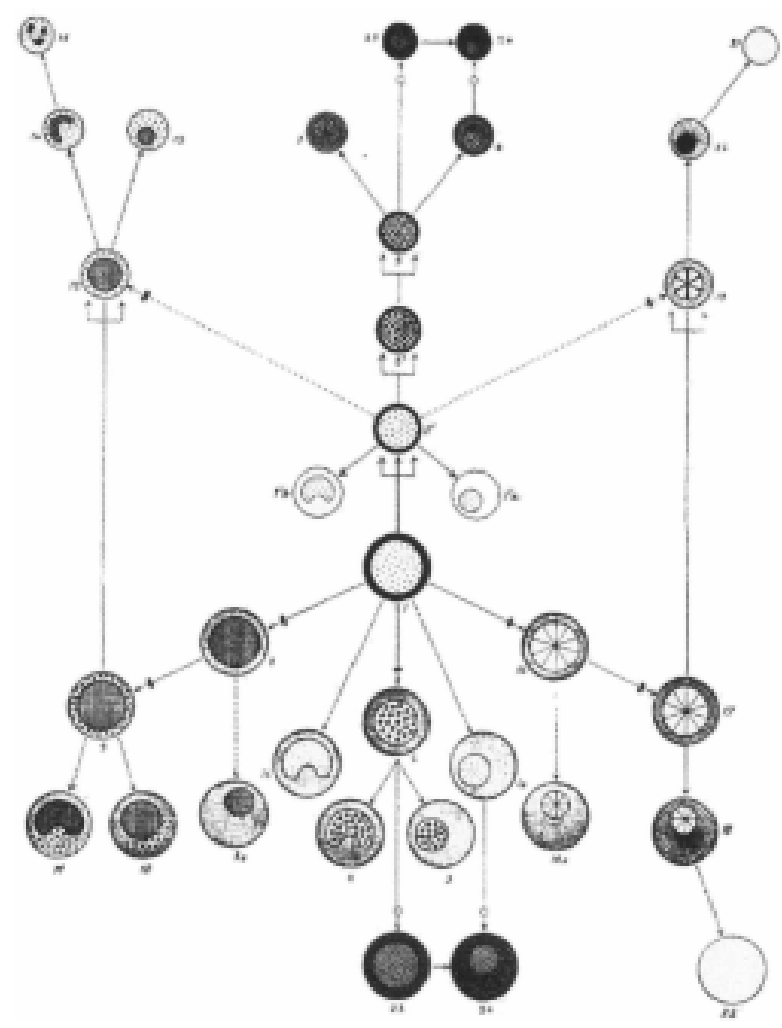

(b)

Figure 2: Stem cells as a constrained space of tree topologies. Bottom: blood stem cell tree, from Pappenheim 1905, 347. 
from the Greek enkaptein, meaning 'encapsulation'. An enkaptic system is comprised of multiple systems, ordered by complexity, such that systems of higher complexity encapsulate ('enclose,' 'have imbedded in them') systems of lower complexity (Rieppel 2009, 313). But the relation between systems is not simply one of containment. Entities composing the different levels of an enkaptic system 'share the fundamental biological property that characterizes all life: the potential to propagate through division' (Rieppel 2009, 313). That is, sub-organismal parts divide, thereby reproducing, and the separated parts are then brought together by being enclosed by the same higher level. The basic mechanisms of enkapsis are division and synthesis, which generate a lineage exhibiting multi-level organization.

Heidenhain proposed enkapsis in the 19 th century as an alternative to reductive conceptions of the organism as an aggregate of cells: 'the organism encapsulates its organs, organs encapsulate their parts, the parts encapsulate cells, cells encapsulate organelles .... The organism thus forms an enkaptic divisional hierarchy ...' (Rieppel 2009, 313-314). Although Heidenhain's concept of development did not include the notion of a stem cell, the lineage view articulated above fits very well with his construal. As discussed above, stem cell lineages generate various aspects of organization that bridge the gap between cells and multicellular organisms. The lineage view of stem cells, in fact, remedies a problem for the original enkaptic view of development. Heidenhain conceived multicellular organisms as enkaptic systems constituted by multiple hierarchical lineages whose components undergo repeated rounds of division and synthesis: cells, organelles, tissues and organs. However, the latter three do not divide within an organism and then synthesize products to form a higher level of organization. The lineage view works better than Heidenhain's original version: stem cell lineages do 'encapsulate' dividing cells, and diverse lineages can be encapsulated together to form tissues and organs. So the view proposed here is very close to Heidenhain's view of the organism, differing mainly in (1) the focus on developmental process rather than organismal structure and (2) the central mediating role of stem cell lineages.

In 20th-century theoretical biology, the concept of enkapsis has been applied to phylogeny rather than ontogeny. However, the theorists that do so continue to rely on cellular analogies, as well as the concept of a 'stem.' For example, Hennig 'was seeking a phylogenetic system in which each group formation relates to a 'beginner' [or 'ancestor']' (Rieppel 2009, 312; quoting Hennig 1965, 99). The origin of a monophyletic taxon is referred to as 'a common stem species' (Rieppel 2009, 314). Hennig's 'deviation rule' states that 'a stem species ceases to exist when it splits into two daughter species, just as a cell ceases to exist when it divides' (ibid, 314). And on Bertalanffy's account 'the two descendant daughter species come together to form a new entity at a higher level of complexity, just as the daughter cells come together to form part of an organ. This new entity that encapsulates the two daughter species is a monophyletic taxon at its lowest level of complexity' (Bertalanffy 1932, 272, quoted in Rieppel 2009, 316). Hennig followed Bertalanffy in taking the encapsulating higher level for species to be the monophyletic taxon, analogous to an organ formed by multiple cells reproduced by division within a lineage.

In at least one respect, however, monophyletic taxa are disanalogous to organs formed from cell lineages. Once 'splitting' has occurred, the new species generated by division need not interact-indeed, lack of such interaction may be a requirement of the species remaining distinct. So it is unclear what relations are involved in repeatedly splitting taxa's becoming unified.12 However, products of cell division within a stem cell lineage do interact with one another and with non-cellular features of their environments. Although much remains unknown

\footnotetext{
${ }^{12}$ It is also difficult to understand what 'interactions among species' (rather than the individuals comprising the relevant populations/species) might amount to.
} 
about normal development in humans and other mammals, some of these interactions are wellcharacterized. If stem cell lineages are enkaptic systems, then the ways that successive generations of progeny cells interact with one another and their environment to produce various aspects of organization are the means by which a new encapsulating level emerges. To understand these means, and thus the cell-organism transition in the process of development for multicellular organisms, we need to look more closely at experiments aimed at characterizing stem cells' developmental potential.

\section{Stem Cell Experiments and Developmental Potential}

The basic design of stem cell experiments is summarized above. But more detail is required to clarify the cell-organism transition. The first quantitative stem cell assay has provided the 'gold standard' for identifying stem cells for over 50 years. The original 'spleen colony assay' detected transplantable cells that form nodule-like colonies on mouse spleen after injection (Till and McCulloch 1961). Each colony is descended from a single cell- the founder cell of the colony. Stem cells are defined as having both self-renewal and differentiation potential. So showing that colony-forming cells are stem cells required 'the demonstration that the colony is transplantable, forming erythroid as well as granulocytic secondary colonies and, which is an even more stringent proof, that it can restore hematopoiesis in a lethally irradiated animal' (Potten and Lajtha 1982, 50). Erythrocytes and granulocytes are mature blood cells with distinct functions. So the appearance of these differentiated cell types in a spleen colony indicates that the founder cell produced these cell types-i.e., that such a cell has blood cell-forming (hematopoietic) potential. Detection of erythrocytes and granulocytes showed that colony-forming (i.e., self-renewing) cells can also give rise to two (or more) blood cell types-they possess at least this differentiation potential. Even stronger evidence ('an even more stringent proof'), however, is that spleen colony-forming cells can, if injected into a mouse with blood and immune cells destroyed by radiation, restore those cells so the animal survives.

That is, the best evidence of HSC capacities is functional regeneration of the blood and immune system in a whole organism (a mouse, in this case). As HSC were the first stem cells that could be quantitatively measured and thus precisely characterized, the experimental standard for determining HSC developmental potential has been applied, with modifications, to all other stem cell types. For ethical reasons, though, the whole-organism 'gold standard' cannot be met for human stem cells. Instead, methods for identifying stem cells in our species use proxies for the whole organism. The methods for producing these proxies reveal important aspects of multicellular organismal organization. The two most common proxies are embryoid bodies and teratomas. Recently, for example, the Progenitor Cell Biology Consortium of the National Heart, Lung and Blood Institute published a standardized 'analytical methodology' for characterizing induced pluripotent stem cells (iPSC), which used both these experimental products to test for stem cell potential (Salomonis et al. 2016). 13 In addition, two new kinds of experiment shed further light on stem cell developmental potential and relations between cell and organism 'levels' in development. I discuss these established methods and new techniques in turn.

\footnotetext{
${ }^{13}$ Disturbingly, the results indicated that the teratoma assay is not a good indicator of stem cell quality (genetic stability).
} 


\subsection{Embryoid bodies}

Cultured pluripotent stem cells typically grow (self-renew) on a two-dimensional solid surface (a culture dish or layer of 'feeder' cells), on which they divide to form colonies. But if suspended in fluid, the cells organize to form 'embryoid bodies': spheres $\sim 0.1-0.2 \mathrm{~mm}$ in diameter, consisting of an inner core of undifferentiated cells wrapped in a layer of more differentiated cells. These simple cellular structures resemble an early stage of mammalian embryonic development, at which embryos are organized into three germ layers with characteristic developmental fates: an outer layer of ectoderm, which gives rise to nervous tissue; an inner layer, endoderm, which gives rise to major organs; and mesoderm in between, which gives rise to muscle, bone, and fat. Cells from embryoid bodies show patterns of developmental potential similar to germ layers, with inner and outer cells 'committed' to different fates. So formation of embryoid bodies is taken as a proxy for the ability to give rise to cells of all three germ layers of a whole organism.

\subsection{Teratomas}

The teratoma assay is performed by injecting human stem cells into a strain of inbred mouse that does not reject the cells, but allows them to grow as tumors within the mice. Resulting tumors are collected, dissected, and histologically analyzed to see if they contain cells of all three germ layers. If so, then scientists conclude the transplanted stem cells are pluripotent. The teratoma assay is actually a modified form of the first experimental test for pluripotency, dating from the 1950s. The first pluripotent stem cells discovered are a form of cancer: teratocarcinoma. This cancer manifests as tumors containing diverse cell types: fat, muscle, skin, nerves, etc.- - even teeth. Teratocarcinoma development was accordingly conceived as a simplified version of embryonic development. 14 The main contrast between the two involves spatio-temporal organization. Teratocarcinomas 'appear to recapitulate many of the events that occur during early embryonic development but in a disorganized manner' (Andrews 2002, 406). Like early embryos, undifferentiated teratocarcinoma cells give rise to a wide range of cell types, including products from each of the three germ layers (ectoderm, mesoderm, and endoderm). In teratoma experiments, cultured cells are transplanted into inbred mice, where they develop into a nonmalignant version of teratocarcinoma. If the resulting tumors contain cells from all three germ layers, albeit disorganized and jumbled, then scientists conclude the transplanted stem cells are pluripotent. As with embryoid bodies, three germ layers in a tumor are considered a proxy for a whole organism. 15

\subsection{Organoids}

Organoids are a new kind of biological entity, produced by manipulating the environment of stem cells and their descendant cells. Lancaster and Knoblich (2014) define an organoid as 'a collection of organ-specific cell types that develops from stem cells or organ progenitors and selforganizes through cell sorting and spatially restricted lineage commitment in a manner similar to in vivo.' That is, organoids are results of in vitro developmental processes which are (i) initiated by stem cells, (ii) mimic or 'recapitulate' the cell-level processes of normal development, and (iii) produce multiple organ-specific cell types which are organized to perform at least one organ-

\footnotetext{
${ }^{14}$ See Fagan 2013a, Chapter 7, for details.

${ }^{15}$ The teratoma assay inversely recapitulates the history of stem cell culture; the first pluripotent stem cells to be experimentally characterized were a transplantable form of cancer (see Fagan 2013a, Chapter 7).
} 
specific function (e.g., neural activity, excretion, contraction). Human organoids have been generated for optic cup, brain, intestine, liver, kidney, stomach, pancreas and more. 16 These 'model organs' are produced by placing stem cells in a three-dimensional spatial arrangement constrained by a synthetic 'scaffold,' in culture media containing biochemical factors that mimic signals for normal cell development. Stem cells under these conditions differentiate to yield more mature cells that then interact to produce an approximation of an organ (Figure 3).

Organoids differ from other in vitro stem cell products, in that they 'self-assemble to form the cellular organization of the organ itself' (Lancaster and Knoblich 2014, 283). This selfassembly is thought to involve two processes: 'cell sorting out' and 'spatially restricted lineage commitment' (ibid). Cells 'sort out' according to adhesion between matching surface molecules, leading to a thermodynamically stable pattern. 'Lineage commitment' refers to the developmental transition from a stem state with the potential to differentiate into various cell types to a state of more restricted potential. The core idea behind organoid self-organization is that the three-dimensional scaffold constrains 'cell fate' (i.e., which differentiation pathway is realized, of multiple possibilities) by structuring cell-cell interactions. In this artificial, scaffolded environment, diverse factors interact dynamically with one another and with stem cells at multiple spatio-temporal scales: 'support cells' secreting signal factors, the extra-cellular matrix (ECM), mechanical forces, oxygen levels, $\mathrm{pH}$, and more (Yin et al. 2016, 28).

Due to the complexity of these dynamic interactions, organoid development is not as robust as normal development. '[E] ach organoid is unique' with respect to the relative positioning of tissue regions, and 'pure populations of single cell types' are not reliably generated by this method (Lancaster and Knoblich 2014). Instead, cell identity in organoids is (largely if not entirely) a result of interactions within a specific dynamic niche (micro-environment). Despite the need for 'exogenous' biochemical factors and the diverse contributing features of the dynamic micro-environment, the capacity to produce organoids is attributed to stem cells' self-organizing potential: 'stem cells exhibit an intrinsic ability to assemble into complex structures' (Yin et al. 2016, 25).

\subsection{Embryonic spatial patterning}

One feature of embryonic organization that organoids lack is the basic polarity of a body plan: dorsal/ventral or anterior/posterior axes. Such polarity is exhibited, however, by pluripotent human stem cells under other conditions. If deprived of biochemical signals needed to maintain self-renewal, and instead provided with molecules that stimulate differentiation in normal development and simple spatial constraints, cultured embryonic stem cells can 'replicat[e] embryonic spatial ordering in vitro'. Significantly, the resemblance is not to very early embryos, the sources from which ESC are derived, but rather embryos up to and after gastrulation (Warmflash et al. 2014, 847). Gastrulation, in normal development, is the stage at which cells are committed to form a single multicellular organism ('individuation'), the three germ layers are distinguished, and generic cell fates are fixed. Currently, there are two different methods for generating embryo-like structures from stem cells: one for mouse, and one for human, ESC. In the mouse procedure, ESC are placed in liquid culture to form embryoid bodies, which are then exposed to a few specific molecules involved in normal development. 17 In this context, cells of the embryoid body undergo a robust differentiation process, culminating in 'polarized

\footnotetext{
${ }^{16}$ Mouse, cancer, and patient-specific organoids have also been produced; see Lancaster and Knoblich 2014 for details and references.

${ }^{17}$ The molecules are activin and Wnt.
} 


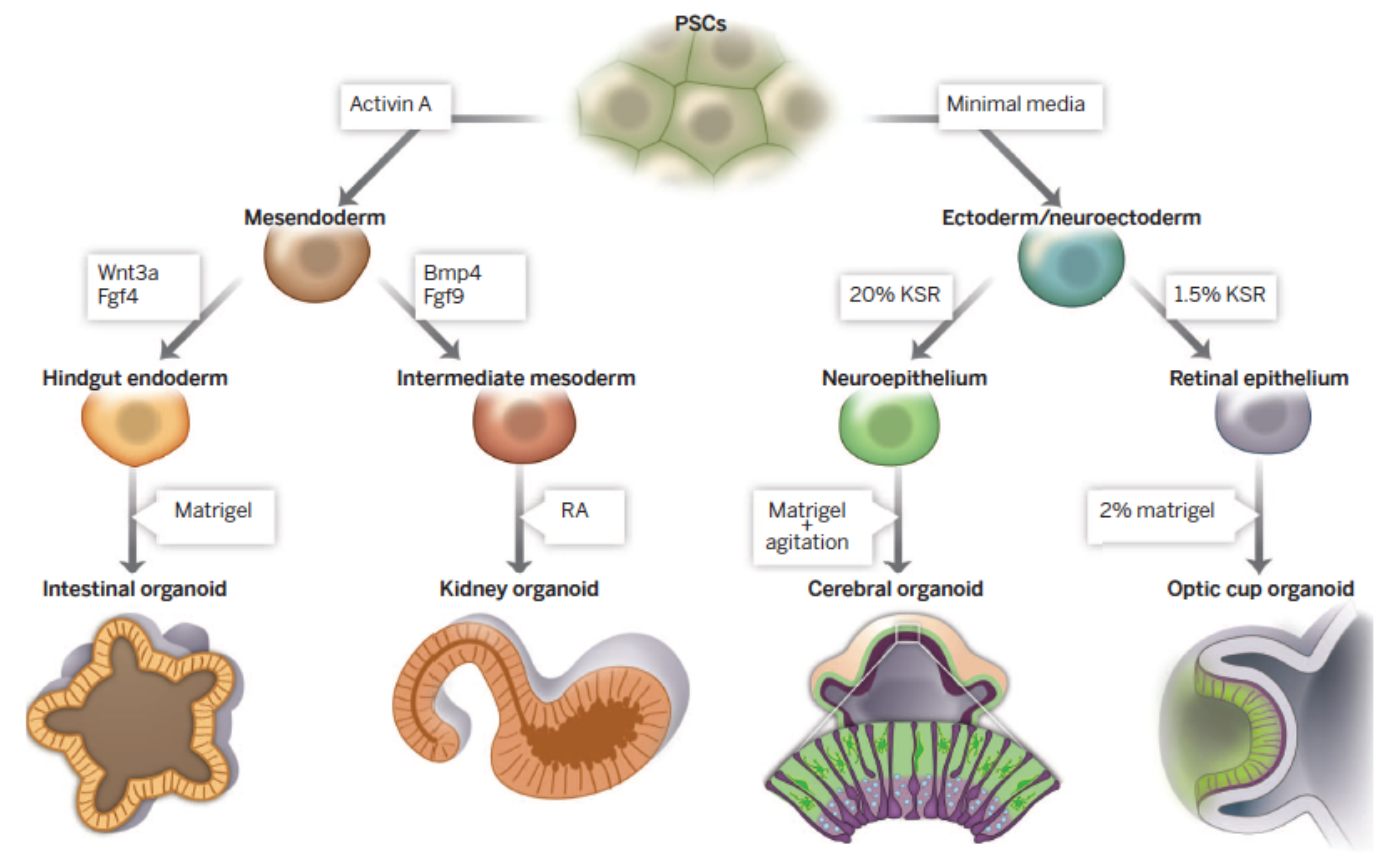

(a)

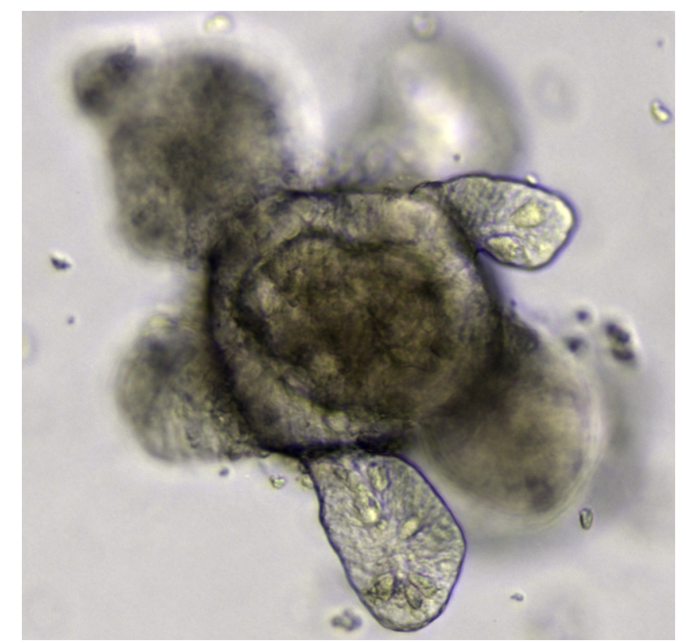

(b)

Figure 3: (a) Schematic of the generation and structure of various organoids (reprinted from Lancaster and Knoblich 2014, Figure 3, 1247125-4, by permission of the American Association for the Advancement of Science). (b) Intestinal organoid (from St Johnston, 2015). 


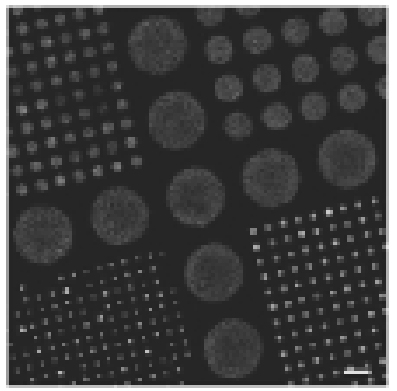

Figure 4: Human embryonic stem cells (hESC) growing in micro-patterned cell culture. (Reprinted from Warmflash et al. 2014, 848, Figure 1b, with permission from Nature Publishing Group.)

structures ... [that] contain cell lineages that are characteristic of the three classic embryonic germ layers: ectoderm, mesoderm and endoderm' (Pera et al. 2015, 917). Although these cellular entities do not resemble embryos anatomically, they 'recapitulate at least some aspects of the spatial organization of the embryo during and after gastrulation' (ibid). More specifically, the self-organizing 'quasi-embryos' exhibit the same repertoire of cell types, which appear in the same order as in normal mammalian development.

The human ESC protocol uses a two-dimensional surface, confining the self-renewing stem cells to small circular areas and then adding a factor involved in normal differentiation (BMP4). In this environment and spatial configuration, hESC differentiate in a 'center-to-periphery' pattern, with 'stemness' remaining highest at the center, declining toward the periphery (Figure 4). The result is a reproducible arrangement of cells resembling the classic germ layers, with ectoderm innermost, surrounded by a ring of mesoderm, and endoderm comprising the outer layer (Pera et al. 2015, 917-918). 18 This simple radial structure does not, of course, resemble an embryo anatomically; nor does the result of the mouse ESC protocol (although the latter are more embryo-like, in being three-dimensional). But these stem cell-derived systems do exhibit several aspects of embryonic organization, including: robust timing and order of appearance of cell types, a reproducible spatial arrangement of germ layers, and polarity along the radial axis. The last of these is demonstrated by varying the size of the circles to which hESC are confined: 'as colony size is reduced, the central fates [cell types to which ESC differentiate] are lost' (Warmflash et al. 2014, 847). This is taken to show that control of cell fate is localized to the ESC colony border, establishing a radial axis for the entire structure.

ESC are undifferentiated cells, and those used in these experiments are assumed to be identical 'clones' of one another. 19 The exogenous biochemical signals are added to cell culture media, and so all the cells are exposed to them. Spatial constraints and geometric organization introduce positional differences among the cells, but the robust appearance of differentiated cell types (germ layers) in a particular spatial orientation is attributed to 'the intrinsic tendency of stem cells to make patterns' (Warmflash et al. 2014, 847). So, as in organoid generation, in vitro embryonic development from stem cells is characterized as 'self-organization,' another developmental capacity of (embryonic) stem cells.

\footnotetext{
${ }^{18}$ There are a number of complexities and uncertainties concerning this result, which is to be expected with a new experimental production. I bracket these complications here.

${ }^{19}$ See Fagan 2013a, under review a, for reasons to question this assumption of initial stem cell homogeneity.
} 


\section{Developmental Versatility and Context-Dependence}

To sum up the argument so far: the enkaptic view of development suggests a way to extend the lineage view of stem cells to include the developmental transition from cell to multicellular organism. On the enkaptic view, diverse cell lineages are encapsulated together to form tissues and organs. Experiments aimed at characterizing stem cell differentiation potential specify the means by which this encapsulation occurs. Embryoid bodies, teratomas, homogeneous populations of differentiated cells, organoids, and spatially patterned embryo-like entities are all produced by specific interactions among cells generated within a stem cell lineage, in conjunction with specific features of the biochemical and physical/geometric environment. So enkapsis is vindicated as an extension of the lineage view of stem cells, by current practices in stem cell research. Although theoretical biologists have heretofore applied the notion of enkapsis to phylogeny rather than to ontogeny, the latter is well-supported by experimental practices while the former is highly contentious. ${ }^{20}$ It remains to be seen how far the analogy between ontogeny and phylogeny, with respect to enkapsis, holds. That project is a task for another paper. Here, I discuss the ontological significance of the experimental methods from the previous section, in particular their bearing on the cell-organism transition and the concept of biological organismality.

The ability to produce parts, or in the extreme case the whole of, an entire multicellular organism, is an essential feature of stem cells. But stem cells vary considerably in their developmental potential - as well as other features, corresponding to variables in the lineage model presented above (Section 2). Pluripotent stem cells, by definition, can give rise to all the main cell types of the body, including the germline. In mice, this capacity has been demonstrated: ESC can give rise to all the cells in a single multicellular organism. Stem cells with more restricted potential (e.g., multipotent) can produce an entire functional organ or tissue within a whole animal. ${ }^{21}$ While teratoma assays also use whole animals, in these experiments the animal is essentially a permissive environment for growth of benign tumors, the products of stem cell differentiation. In vitro stem cell experiments also use proxies for a whole animal or a particular organ/tissue: embryoid bodies, organoids, and embryo-like organization. Other studies of stem cells use simpler culture systems, directing differentiation toward a single mature cell type of interest, such as neurons, muscle, or blood. It is tempting to construe these diverse model systems as successive approximations of human embryonic development, with more sophisticated models able to 'forge a better replica of the embryo' or a particular organ (Pera et al. 2015, 918). On this view, the more accurately stem cell experiments mimic the biochemical, physical, and geometric context (environment) of normal in vivo development, the more closely the experimental products resemble an embryo.

While this progressive ideal for modeling is an important part of stem cell reseachers' motivation, it does not fully capture the results of recent stem cell experiments. The various methods discussed above all realize stem cells' generative capacities, but in different ways. The results do not form a tidy sequence of better and better models. Rather, they exhibit the variety of modes of development that stem cells can initiate. Scientists' own accounts reinforce this idea.

\footnotetext{
${ }^{20}$ Contra Bertalanffy: 'Such unification [the result of enkapsis] becomes increasingly tighter, the component parts hence increasingly more differentiated and less independent, the further one ascends in the ontogenetic and phylogenetic hierarchy' (Bertalanffy 1932, 272, quoted in Rieppel 2009, 316).

${ }^{21}$ In practice, only blood-forming stem cells (HSC) from particular strains of laboratory mice have been identified and characterized are characterized in this way. But whole animal experiments remain the gold standard for much of stem cell research.
} 
Table 1: Modes of development and aspects of organization to which stem cells contribute.

\begin{tabular}{ll}
\hline Mode of development & Aspects of organization \\
\hline normal & all, complex, robust \\
pathological & specialized cell types, 3D structure, non-robust \\
simplified & generic cell types, simple 3D structure \\
cellular & specialized cell types \\
organ-like & specialized cell types, complex 3D structure, non-robust \\
embryo-like & generic cell types, simple 2D structure, polarity \\
\hline
\end{tabular}

One influential recent review characterizes teratomas, embryoid bodies, and organoids as three distinct 'paradigms of self-organization' from stem cell research (Lancaster and Knoblich 2014). Another uses diagrams to indicate the 'parallel tracks' of normal in vivo development, in vitro stem cell differentiation, teratomas, and organoids (Yin et al. 2016, 27). Rather than falling on a single continuum, these diverse methods and results form an array of modes of development emanating from stem cells.

At least six such modes can be distinguished (Table 1). First, in normal development, a single cell in an appropriate environment transforms into a viable multicellular organism. Stem cells can contribute to this process, as whole-animal experiments (with mice) demonstrate. Unmanipulated normal development can be reasonably assumed to involve a variety of selfrenewing and differentiating stem cells as well. Stem cells isolated from specific organs or tissues in mature organisms (i.e., 'adult stem cells') are the 'relict populations' of the more comprehensive generative process of stem cell-mediated normal development. Normal development proceeds via intricate regulation of the sizes of cell populations arranged in developmental hierarchy, knitted together via cell signaling pathways.

Second, teratoma formation is a pathological counterpart of normal development. Stem cells that produce teratomas do so only in the peculiarly permissive environment of an immunodeficient mouse, of a strain that is prone to forming tumors. Under these (highly artificial) conditions, pluripotent stem cells give rise to representatives of the full range of specialized cell types in the human body-cells of all three germ layers. The malignant form of teratoma is teratocarcinoma: a cancer arising in mammalian germ cells. Teratocarcinoma produces tumors comprised of a disturbing mélange of specialized cells and tissues: teeth, hair, muscle, etc. Their malignancy consists in sustained self-renewal of undifferentiated cancer cells, which give rise to the more specialized cells and tissues comprising the bulk of the tumor. Generalization of this mode of development to other cancers is the 'cancer stem cell model' (Laplane 2015; Fagan 2016c).

Third, embryoid bodies are produced via a simplified, minimal mode of development. Specialized cells appear, arranged into a spherical structure resembling an early embryo-but drastically simplified, with just inner and outer layers. The environment for producing embryoid bodies is similarly minimal: ESC are detached from a two-dimensional surface, where they divide to form sheet-like colonies, and placed in liquid media. This new physical context induces ESC to form spheres with a robust inner/outer arrangement. As in normal development, position in this structure predicts cell fate: inner- and outer-layer cells give rise to different mature cell types. This is ascertained by changing cells' environment yet again: cells from an embryoid body are distributed to different two-dimensional culture environments, each designed to encourage differentiation into a particular specialized cell type, such as muscle, blood, or neuron. 
Fourth, 'directed differentiation' experiments establish two-dimensional sheet-like populations of particular mature cell types. Considered collectively, these experiments are another way to demonstrate stem cell potential, or potency. Stem cell potential is usually conceived in terms of the number of cell types comprising the body of a multicellular organism. Directed differentiation experiments are used throughout stem cell research, to show that a given stem cell's potential includes the cell type of interest, and/or to produce pure populations of that cell type. These represent a fourth mode of development, restricted to the cellular level. In this mode, development from stem cells to each specialized cell type is achieved separately, using a distinct culture environment.

Organoids and embryonic spatial patterning highlight a fifth and sixth mode of development: organ-like and embryo-like, respectively. Environments that produce embryo-like spatial patterning or organoids contain specific biochemical signals and are structured in two or three dimensions, respectively. The results of development in these structured environments are more elaborate than other stem cell-derived experimental products. Organoids are composed of multiple cell types, arranged to morphologically and functionally resemble a particular organ. Organoids reveal another aspect of developmental potential; the 'self-organizing properties of stem cells' (Lin et al. 2016, 25; italics in original). These properties connect cell- and tissue/organ levels of biological organization. Organoids are model systems that aim at 'multi-level recapitulation of the body' (Yin et al. 2016, 25). Embryo-like spatial patterning approximates a post-implantation embryo in another way, exhibiting robust periphery-to-center polarity that resembles the rudiments of a body plan.

Apart from normal development, each mode highlights different aspects of the organization involved in generating a multicellular organism from undifferentiated cells (Table 1). Pathological development yields specialized cells and tissues in a three-dimensional arrangement, but the resulting teratomas (and other cancers) are 'disordered' in at least three respects. First, the set of specialized cells and tissues that appear varies across tumors; pathological development is not robust, on a cellular level. Second, the specialized cells and tissues that appear are not arranged into functional organs; this mode of development is not robust at the organ level either. Finally, the specialized cells and tissues of a teratoma are not arranged so as to constitute an autonomous organism; robustness is lacking at the organism level. Embryoid bodies, in contrast, show robust arrangement of differentiated cells in a three-dimensional structure that resembles an embryo. This mode of development is simplified and minimal, rather than unpredictably disordered; embryoid bodies are robustly organized at cell and organism levels. However, their organization is extremely simple, lacking mature cells and any distinct organs.

Directed development, considered as a collection of experiments, can produce (nearly) the full range of mature specialized cells that make up an organismal body. But this is a fragmented, spatio-temporally dispersed reflection of normal development. Many specialized cell types can be robustly produced as pure populations, from two-dimensional stem cell cultures. But other aspects of normal development are lacking. The result of directed differentiation experiments, considered collectively, is a thorough disarticulation of organismal organization, into homogeneous populations of specialized cell types.

Organoids help bridge the gap between cell- and organism-level organization. The combination of three-dimensional scaffolds and specific signals produces multiple specialized cell types that approximate the three-dimensional and functional organization of a particular organ. Although positioning of particular cell types within an organoid is not robust, their development preserves many organizational features of normal organ development. Finally, spatially patterned embryo-like structures exhibit a simplified body-plan, with robust organization of 
different cell types characteristic of post-implantation human embryos.

Each of these six modes of development, with their different aspects of organization, is initiated by stem cells. Stem cell potential, as noted above, is typically defined in terms of the range of cell types that a given stem cell can produce, if placed in the appropriate environment. But that concept of potential does not capture the diverse organizational capacities revealed by experiments using the different developmental modes. Experimental practices, considered collectively, show that stem cells are capable not only of differentiating to produce a particular range of cell types, but of realizing different processes or modes of differentiation. I propose the term 'developmental versatility' to refer to this capacity: the ability to produce different aspects of organization associated with different modes of development: normal, disordered, simple, cellular, organ-like, and embryo-like. The transformation of a developing biological individual from a single cell to a mature multicellular organism proceeds by means of stem cells' enkaptic lineages, which realize some or all of these diverse modes of development.

Experimental practices of stem cell research reveal a further result as well. Which mode or set of modes of development relating cell to organism is realized in any particular developmental process initiated by a stem cell depends on features of that cell's environment, and of its descendants' environment. These features include the presence or absence of specific biochemical signals, spatial/geometric arrangement of cells, physical boundaries of the system, and physical factors such as oxygen levels or $\mathrm{pH}$. The environmental dependence of modes of development initiated by stem cells is a special case of a more general conclusion: stem cell identity is context-dependent.22 Experiments that reveal developmental potential make use of this feature, inducing stem cells to differentiate in specific (and diverse) ways by manipulating the geometry, biochemical composition, or cellular makeup of their environment. In the best-characterized in vivo stem cell systems, such as mouse intestinal epithelium and fly testes, stem cell capacities are dependent on a specific position within the relevant organ (Barker et al. 2012; Cherry and Matunis 2010). Given these spatial constraints, cell division allows only a few cells to remain in 'a stem cell permissive environment' (or niche), while other progeny are expelled and perforce differentiate (Barker et al. 2012, 452-453). 23]

Furthermore, the reverse is possible: given the right environmental conditions, at least some non-stem cells can transform into stem cells. The most celebrated example of this is 'reprogramming,' a method by which differentiated cells of various types are transformed (albeit with a very low success rate) to 'induced' pluripotent stem cells (iPSC; Takahashi and Yamanaka 2006). The transformation is accomplished by altering the expression of a few specific genes in cultured cells extracted from an organism. After a few weeks of in vitro growth with altered gene regulation, a few cells change their appearance and molecular traits so as to resemble embryonic stem cells, which can then in turn be 'directed' to differentiate along various pathways. Other experimental reversals of cell development are possible as well. It has long been recognized that the 'stem cell phenotype appears to be by no means hard-wired,' but rather 'the behavior of stem cells is controlled by the microenvironment' (Potten and Lajtha 1982, 454; Yin et al. 2016, 27; see also Barker et al. 2012, 454). Context-dependence of stem cell capacities is such that processes of development initiated by stem cells are in principle reversible. This experimental discovery is a profound alteration of traditional ideas about the process of biological development, the full

\footnotetext{
${ }^{22}$ For other lines of argument for this conclusion, and the related claim that stem cells are 'experimentally relative,' see Fagan 2013a, Chapter 3, 2013b, 2016b.

${ }^{23}$ The precise nature of the hematopoietic stem cell (HSC) niche is a matter of long-standing controversy. However, it is widely accepted that the bone marrow microenvironment plays a role in the balance of HSC self-renewal and differentiation (Sean Morrison 2016, pers. comm.).
} 
implications of which have not yet been worked out even by the researchers involved.

This motivates another extension of the lineage view. To accommodate stem cells' environmental sensitivity, stem cell lineage trees should be conceptualized in association with one or more specific environments $\left(E_{1 \ldots n}\right)$. Applied to a broad range of experimental results, this framework could reveal systematic patterns in stem cell development with respect to particular environmental factors. Factors of interest could then be associated with a set of tree topologies, allowing for new comparisons and insights to emerge. However, the main point for the purpose of this paper is that context-dependent stem cell development has ramifications for concepts of biological individuality and organismality. The latter notion, in particular, is often understood as requiring internal directedness and autonomy. ${ }^{24}$ The methods and results of stem cell experiments show that such requirements need to be re-examined. Stem cells exhibit not only developmental potential, the ability to generate one or more differentiated cell types, but also developmental versatility, the capacity to produce one or more modes of organismal organization. The 'flip-side' of developmental versatility is context-dependence: which modes of organismal organization are realized in a particular developmental process depends on the specific combination of environmental factors present. Over 50 years of stem cell experiments have taught us how to 'steer cell fate through exogenous niche components'- that is, to control stem cell capacities through features of cells' environments. ${ }^{25}$ Although this control is imperfect owing to the many gaps in our knowledge of stem cell development, and intra-cell regulation of gene expression is of course also crucial, decades of experiment have shown that stem cell identity is exquisitely sensitive to features of the local microenvironment, both in vivo and in vitro. Manipulating stem cells' context-dependence is the basic design principle of all stem cell experiments: candidate stem cells are moved to a new environment, and their or their descendants' characteristics in that context are measured. This basic experimental strategy continues to be used, revealing piecemeal the diverse factors involved in generating organismal organization from a stem cell. Development, including the transformation from cellular to organismal levels of organization, is not an ineluctable, internally-driven process. Biological individuals that develop do so as part of a dynamic assemblage of interacting factors, including features of the environment as well as cells 'stemming' from a single lineage.

\section{Conclusion}

This paper has motivated and defended the lineage view of stem cells as a conceptual framework, i.e., an ontology, for understanding the cell-organism relation in biological development. I have argued that this relation is fruitfully conceived in terms of cell lineages that form an enkaptic system generated by a single stem. According to the basic lineage view, a stem cell is the starting point of a cell lineage with a specific organismal source, time-interval of existence (measured in cell divisions), and 'tree topology' of branch-points linking the stem to developmental termini. Refinement of this basic picture is necessary, to account for the cell-organism transition in the process of development. Hennig's conception of enkapsis provides the needed refinement. Further explication of the ways in which cells interact with one another and with features of their environment, thereby giving rise to tissues, organs, and the whole organism, is provided by examination of experimental methods used to determine stem cells' developmental potential. These various methods produce new artificial entities which reveal aspects of multicellular or-

\footnotetext{
${ }^{24}$ I discuss these ideas further in Fagan (under review a).

${ }^{25} \mathrm{~A}$ stem cell's local environment is extremely dynamic, and stem cells influence and are influenced by it. So stem cell capacities are realized via an intricate interplay of cell and biochemical interactions.
} 
ganization: cultured cells from 'direct differentiation,' embryoid bodies, teratomas, organoids, and embryo-like structures. The different modes of development associated with these methods indicate the different aspects of organization (simple, dispersed, cellular, organ-like and embryolike) which are integrated in the overall process of normal organismal development. As research on the experimental systems described above goes forward, the expanded lineage view of stem cells can serve as a unifying framework for coordinating these results into an explicit ontology of biological development. But even lacking such a comprehensive framework, some ontological conclusions can be drawn. Arguably the most significant of these is the context-dependence of developmental processes involving stem cells_-including in principle reversibility.

\section{Acknowledgments}

Thanks to Thomas Pradeu for the invitation to contribute an essay to this Special Issue. This paper has benefited from discussions with Louiseneh Arakelian, Julia Bursten, Sara Green, Matt Haber, Lucie Laplane, Anne Peterson, Thomas Pradeu, and Joe Rouse, and from comments and questions by audiences at the University of Utah (April 2016), the IHPST workshop on Cancer and Philosophy (Paris, April 2016), the University of Bordeaux (May 2016), and the Unnatural Kinds workshop (San Francisco State University, May 2016). Lucie Laplane, the editors, and an anonymous reviewer provided helpful comments on an earlier draft. Funding was provided by the Humanities Research Center at Rice University, the Mosle Research Foundation, the University of Utah College of Humanities, and a generous donation from the family of Sterling M. McMurrin.

\section{Literature cited}

Andrews, P. 2002. From Teratocarcinomas to Embryonic Stem Cells. Philosophical Transactions of the Royal Society of London, Series B 357: 405-417.

Barker, N., van Oudenaarden A., Clevers H. 2012. Identifying the Stem Cell of the Intestinal Crypt: Strategies and Pitfalls. Cell Stem Cell 11: 452-460.

Bertalanffy, L. 1932. Theoretische Biologie, Band I. Gebr Bornträger, Berlin.

Bouchard, F. and P. Huneman, editors. 2013. From Groups to Individuals: Evolution and Emerging Individuality. Cambridge: MIT Press.

Cell Therapy and Regenerative Medicine Glossary. 2012. Stem cell. Regenerative Medicine 7: S1S124.

Chen, R.-L., O. Bueno, and M. B. Fagan, editors. under review. Individuation across Experimental and Theoretical Sciences. Under contract with Oxford University Press.

Cherry C., and E. Matunis. 2010. Epigenetic Regulation of Stem Cell Maintenance in the Drosophila Testis via the Nucleosome-Remodeling Factor NURF. Cell Stem Cell 6: 557-567.

Clermont, Y., and C. P. Leblond. 1959. "Differentiation and Renewal of Spermatogonia in the Monkey, Macacus Rhesus." American Journal of Anatomy 104: 237-273.

Dröscher, A. 2014. "Images of Cell Trees, Cell Lines, and Cell Fates: The Legacy of Ernst Haeckel and August Weismann in Stem Cell Research." History and Philosophy of the Life Sciences 36: 157186.

Dupré, J. 2012. “The Polygenomic Organism.” In Processes of Life: Essays in the Philosophy of Biology, 116-127. Oxford: Oxford University Press. 
Dupré, J., and Maureen O’Malley. 2009. “Varieties of Living Things: Life at the Intersection of Lineage and Metabolism.” Philosophy and Theory in Biology 1:e003.

European Stem Cell Network. 2016. “Stem Cell Glossary.” http://www.eurostemcell.org/ stem-cell-glossary/\#letters.

Evans, M., and M. Kaufman M. 1981. "Establishment in Culture of Pluripotential Cells From Mouse Embryos.” Nature 292: 154-156.

Fagan, M. B. 2013a. Philosophy of Stem Cell Biology: Knowledge in Flesh and Blood. London, PalgraveMacmillan.

Fagan, M. B. 2013b. "Philosophy of Stem Cell Biology: An Introduction." Philosophy Compass 8: $1147-1158$.

Fagan, M. B. 2016a. "Cell and Body: Individuals in Stem Cell Biology." In Individuals Across the Sciences, edited by T. Pradeu and A. Guay, 122-143. Oxford University Press.

Fagan, M. B. 2016b. "Generative Models: Human Embryonic Stem Cells and Multiple Modeling Relations." Studies in History and Philosophy of Science 56: 122-134.

Fagan, M. B. 2016c. "Pathways to the Clinic: Cancer Stem Cells and Challenges for Translational Research.” To appear in Philosophy of Molecular Medicine, edited by M. Nathan and G. Bonniolo. Routledge.

Fagan, M. B. under review a. "Stem Cell Biology: A Conceptual Overview." for Stem Cell Research: An Interdisciplinary Discussion of the Current Challenges and the Perspectives for Philosophical Ethics, edited by C. Hauskeller, A. Manzeschke, and A. Pichl.

Fagan, M. B. under review b. "Individuality, Organisms, and Cell Differentiation." To appear in Individuation across Experimental and Theoretical Sciences edited by R.-L. Chen, O. Bueno, M. B. Fagan. Oxford: Oxford University Press.

Godfrey-Smith, P. 2009. Darwinian Populations and Natural Selection. Oxford: Oxford University Press.

Haber, M. H. 2012. "Multilevel Lineages and Multidimensional Trees: The Levels of Lineage and Phylogeny Reconstruction.” Philosophy of Science 79: 609-623.

International Society for Stem Cell Research. 2016. Stem cell glossary. http://www.isscr.org/ visitor-types/public/stem-cell-glossary/\#stem.

Lajtha, L. G. 1979. “Stem Cell Concepts.” Differentiation 14: 23-34.

Lancaster, M. A., and J. A. Knoblich. 2014. "Organogenesis in a Dish: Modeling Development and Disease Using Organoid Technologies.” Science 345: 124-125.

Laplane, L. 2015. Cancer Stem Cells: Ontology and Therapies. Cambridge: Harvard University Press.

Melton D. 2013. "Stemness: Definitions, Criteria, and Standards." In Essentials of Stem Biology, 3rd edition, edited by R. Lanza, and A. Atala, 7-17. San Diego, CA: Academic Press.

Minelli, A. 2011. "Animal Development, an Open-Ended Segment of Life." Biological Theory 6: 4-15.

Minelli A., and T. Pradeu, editors. 2014. Towards a Theory of Development. Oxford: Oxford University Press.

National Institutes of Health. 2015. "Glossary." In Stem Cell Information [World Wide Web site]. Bethesda, MD: National Institutes of Health, U.S. Department of Health and Human Services. [cited March 13, 2016] Available at http://stemcells.nih.gov/glossary/Pages/Default.aspx

O’Malley, M. 2014. Philosophy of Microbiology. Cambridge: Cambridge University Press. 
Pappenheim, A. 1905. "Bemerkungen über artliche Unterschiede und die gegenseitigen genetischen Beziehungen zwischen den verschiedenen lymphoiden Zellformen des Blutes.” Folia haematologica 9: 321-404.

Pera, M. F., G. de Wert, W. Dondorp, R. Lovell-Badge, C. L. Mummery, M. Munsie, and P. P. Tam. 2015. "What if Stem Cells Turn Into Embryos in a Dish?" Nature Methods 12: 917-919.

Potten, C. S., and L. G. Lajtha. 1982. "Stem Cells Versus Stem Lines." Annals of the New York Academy of Sciences 397: 49-61.

Pradeu, T. 2012. The Limits of the Self: Immunology and Biological Identity, translated by Elizabeth Vitanza. New York: Oxford University Press.

Pradeu, T. 2016. “Thirty Years of Biology \& Philosophy: Philosophy of Which Biology?” Biology and Philosophy. doi:10.1007/s10539-016-9558-7.

Pradeu, T., L. Laplane, M. Morange, et al. 2011. "The Boundaries of Development.” Thematic Section. Biological Theory 6: 1-102.

Pradeu, T., G. Kostyrka, and J. Dupré. 2016. "Understanding Viruses: Philosophical Investigations.” Studies in History and Philosophy of Biological and Biomedical Sciences 59: 57-63.

Rieppel, O. 2009. "Hennig's Enkaptic System." Cladistics 25: 311-317.

Salomonis, N., P. I. Dexheimer, L. Omberg, R. Schroll, S. Bush, J. Huo, et al. 2016. "Integrated Genomic Analysis of Diverse Induced Pluripotent Stem Cells From the Progenitor Cell Biology Consortium." Stem Cell Reports 7: 110-125.

St Johnston, D. 2015. “The Renaissance of Developmental Biology.” PLoS Biol 13: e1002149. doi:10.1371/journal.pbio.1002149.

Stem Cell Basics. 2015. In Stem Cell Information [World Wide Web site]. Bethesda, MD: National Institutes of Health, U.S. Department of Health and Human Services

Takahashi, S., and S. Yamanaka. 2006. "Induction of Pluripotent Stem Cells From Mouse Embryonic and Adult Fibroblast Cultures by Defined Factors." Cell 126: 663-676.

Thomson, J., J. Itskovitz-Eldor, S. Shapiro, M. Waknitz, J. Swiergel, V. Marshall, and J. Jones. 1998. “Embryonic Stem Cell Lines Derived From Human Blastocysts.” Science 282: 1145-1147.

Till, J., and E. McCulloch. 1961. "A Direct Measurement of the Radiation Sensitivity of Normal Mouse Bone Marrow Cells.” Radiation Research 14: 213-222

Warmflash, A., B. Sorre, F. Etoc, E. D. Siggia, A. H. Brivanlou. 2014. "A Method to Recapitulate Early Embryonic Spatial Patterning in Human Embryonic Stem Cells.” Nature Methods 11: 847854.

Yin, X., B. E. Mead, H. Safaee, R. Langer, J. M. Karp, O. Levy. 2016. "Engineering Stem Cell Organoids." Cell Stem Cell 18: 25-38.

(C) 2017 Author(s)

This is an open-access article distributed under the terms of the Creative Commons AttributionNonCommercial-NoDerivatives 4.0 International license, which permits anyone to download, copy, distribute, or display the full text without asking for permission, provided that the creator(s) are given full credit, no derivative works are created, and the work is not used for commercial purposes.

ISSN 2475-3025 